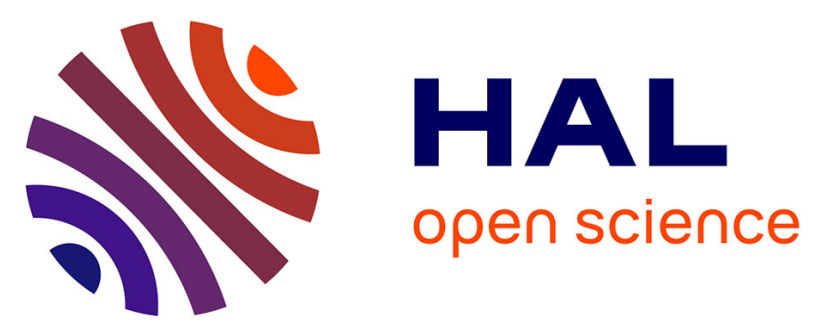

\title{
Improvement of shadowing with iterative physical optics for radiation pattern of mounted antennas
} Antoine Thomet, Gildas Kubicke, Christophe Bourlier, Philippe Pouliguen

\section{To cite this version:}

Antoine Thomet, Gildas Kubicke, Christophe Bourlier, Philippe Pouliguen. Improvement of shadowing with iterative physical optics for radiation pattern of mounted antennas. 16th International symposium on ANtenna Technology and applied ElectroMagnetics (ANTEM 2014), Jul 2014, Victoria (BC), Canada. pp.1 - 2 10.1109/ANTEM.2014.6887701 . hal-00967937

\section{HAL Id: hal-00967937 https://hal.science/hal-00967937}

Submitted on 18 Dec 2015

HAL is a multi-disciplinary open access archive for the deposit and dissemination of scientific research documents, whether they are published or not. The documents may come from teaching and research institutions in France or abroad, or from public or private research centers.
L'archive ouverte pluridisciplinaire HAL, est destinée au dépôt et à la diffusion de documents scientifiques de niveau recherche, publiés ou non, émanant des établissements d'enseignement et de recherche français ou étrangers, des laboratoires publics ou privés. 


\begin{abstract}
The prediction of Radar Cross Section (RCS) of complex targets which present shadowing effects is an interesting challenge. This paper deals with the problem of shadowing effects in the computation of electromagnetic scattering by a complex target using Iterative Physical Optics (IPO). The original IPO is limited to cavities applications, but a generalized IPO can be applied to arbitrary geometries. This paper proposes a comparison between the classical PO approach and a physical approach based on shadow radiation (around forward direction) with PO approximation for the consideration of shadowing effects in generalized IPO. Based on the integral equations, a rigorous demonstration of this physical shadowing is provided. Then simulation results illustrate the interest of using physical shadowing both from the transmitter and towards the receiver, compared to the classical approach.
\end{abstract}

\title{
1. INTRODUCTION
}

Computing electromagnetic signature of complex targets presenting shadowing effects is a complex problem for which many solutions have been proposed. Each of these solutions presents benefits and drawbacks, and two different kinds of methods can be used for arbitrary shaped cavities: rigorous numerical methods and asymptotic methods.

Numerical methods, like Method of Moments (MoM), can be used to calculate RCS (Radar Cross Section) of targets with a good precision. These methods, which do not apply any approximation (but approximation linked to meshing), are known to provide excellent results, but their complexity is high. MoM has a complexity of $\mathcal{O}\left(N^{3}\right), N$ being the number of unknowns (which is equal to the number of non boundary edges of a meshed target). Thus, in case of great target's dimensions (compared to wavelength), these methods are generally not used, due to their computing time and memory requirement. Nevertheless, MoM will be used in this paper as a reference method.

To overcome this issue, asymptotic methods have been developed and can be used in highfrequency domain for arbitrarily shaped targets with a reduced complexity. These methods are based on Geometrical Optics (GO), based on ray trajectories, and/or Physical Optics (PO), using surface currents to calculate scattered fields. When multiple reflections occur, PO is generally preferred to GO, as GO is less precise, particularly in case of highly curved geometries.

Iterative Physical Optics (IPO) [1-3] is an asymptotic method based on PO. The method has been originally developed to calculate RCS of cavities [1] and has been generalized to arbitrary geometries [4]. This method can be described by an algorithm in 4 steps:

1) Iteration 0: this first step is to estimate surface currents (inside the cavity, in the case of original formulation, or on the structure if using generalized IPO) from the incident electromagnetic field;

\footnotetext{
Received 12 February 2014, Accepted 13 July 2014, Scheduled 6 August 2014

* Corresponding author: Antoine Thomet (antoine.thomet@univ-nantes.fr).

${ }^{1}$ IETR (Institut d'Electronique et des Télécommunications de Rennes) Laboratory, CNRS UMR 6164, LUNAM Université, Université de Nantes, La Chantrerie, Nantes 44306, France. ${ }^{2}$ DGA/DT/MI (Direction Générale de l'Armement — Direction Technique — Maîtrise de l'Information), CGN1 division, Bruz 35170, France. ${ }^{3}$ DGA/DS/MRIS (Direction Générale de l'Armement — Direction de la Stratégie - Mission pour la Recherche et l'Innovation Scientifique), Bagneux 92221, France.
} 
2) Field scattering among the structure (Iterative): the calculated currents imply an electromagnetic field scattered towards the whole structure;

3) Updating surface currents (Iterative): the calculated fields imply evolution of the surface currents;

4) Scattering through observation: field scattered towards the observation point is then calculated.

Knowing that surface currents and fields inside the cavity are inducing each other, steps 2 and 3 are computed iteratively. The end of iterations can be either determined by a fix number of iterations [1] or by a convergence criterion $[2,5]$. IPO complexity is $\mathcal{O}\left(K N^{2}\right), K$ being the number of iterations and $N$ the number of sample points. It is important to note that this asymptotic method permits to reduce significantly the complexity, as the number of necessary samples is not the same as in rigorous methods: typically, MoM needs a meshing between $\lambda / 8$ and $\lambda / 10$, when IPO can be run for meshing around $\lambda / 4$ or even $\lambda / 3$ (for example the number of elements in a $\lambda^{2}$ surface is from 64 to 100 for MoM, and reduced to 16 or 9 for IPO).

The first step of IPO, iteration 0, can have a strong influence on the final result, since the higherorder currents calculation depends on it. In $[1,6]$, the authors used an aperture surface to calculate currents inside the cavity. Although it is accurate in case of scattering by cavities, this method is not a general shadowing approach, as it needs to determine the aperture surface of the cavity. This paper focuses on the use of general shadowing approaches in IPO. Note that these techniques, based on shadowing effects between two objects, can be used not only at iteration 0 , but also at steps 4 (when the observer cannot directly see the whole target), and, for complex geometries, at step 3 (when a part of the object's walls is hidden from another part), which is not the case of shadowing by an aperture surface.

Classical PO approach (used for example in [1] in the iterations), considers a shadowed region, on which currents vanish, and a lit region, on which currents are calculated by the PO approximation. The main drawback of this method, detailed in this paper as "geometrical shadowing", resides in the fact that a ray bouncing is used to determine the shadow zone, inducing discontinuous currents.

Another approach, included in particular IPO by Burkholder et al. [7] as part of the first iteration of IPO, is discussed in this paper, and referred as "physical shadowing" (as it is based on physical approach, and not geometrical technique as in the classical IPO method). This technique, based on PO shadow radiation [8-13], is a general method of considering the shadowing effect, which does not need to use ray tracing. To our knowledge, no rigorous proof of this method, or comparison to geometrical shadowing has been published. This paper focuses on the demonstration of the physical shadowing principle, proving that it is a more rigorous approach than the geometrical shadowing.

First, Section 2 presents the theory of both general shadowing approaches. Then simulations are shown in Section 3, using the example of the dihedral reflector: differences in implementation of the methods are explained, then results are presented for different configurations, in order to observe the impact of the shadowing approach considered and to study the improvement of using the physical shadowing in IPO, both at incidence and at observation. Finally, Section 4 gives concluding remarks and prospects.

\section{THEORETICAL STUDY}

\subsection{Fields Integral Equations}

In this paper, unitary vectors will be noted with a hat: $\hat{v}$ or $\hat{V}$, while other vectors will be noted in capital letter with an arrow: $\vec{V}$. $\vec{\nabla}$ stands for nabla vectorial operator. Time convention $e^{-j \omega t}$ is assumed and omitted in the equations.

This part treats on general problem of scattering by a single object [14]. Let us consider the scenario depicted in Figure 1.

An object of volume $V$, bounded by surface $S$ and centered on the phase origin, is surrounded by a propagation medium which volume $V_{\infty}$ is infinite. Propagation volume is noted $V_{0}$, where $V_{\infty}=V_{0} \cup V$. Permittivity and permeability in $V_{0}$ are noted $\epsilon$ and $\mu$, respectively. A mobile point $P^{\prime}$, of coordinates vector $\vec{R}^{\prime}$, is used for integration. When $P^{\prime}$ is located on surface $S$, it is linked to a surface element $d S^{\prime}$, with normal vector $\hat{n}^{\prime}$ pointing outside $V$. On the other hand, when it is located in a volume, a volume 


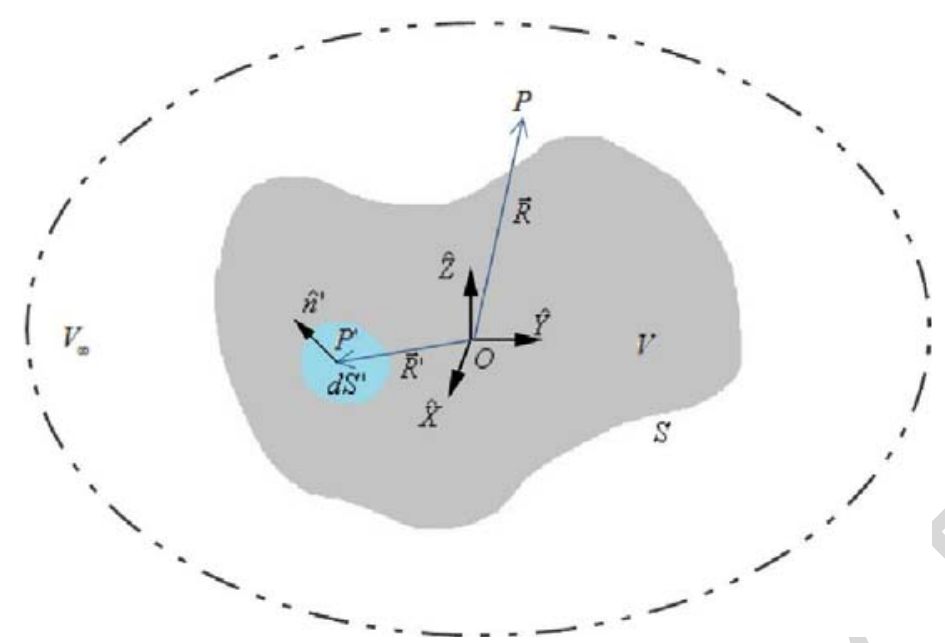

Figure 1. General configuration of scattering problem.

element $d V^{\prime}$ will be associated to $P^{\prime}$. All parameters linked to $P^{\prime}$, that need to be integrated, will be noted with a "'’". Electric and magnetic fields at $P^{\prime}$ will be noted $\vec{E}^{\prime}$ and $\vec{H}^{\prime}$ respectively. Sources are represented, at each point $P^{\prime}$ of $V_{0}$, by electric and magnetic current densities $\vec{J}_{\mathrm{i}}^{\prime}$ and $\vec{M}_{\mathrm{i}}^{\prime}$, and by electric and magnetic charge densities $\rho_{e}^{\prime}$ and $\rho_{m}^{\prime}$. Observation point is called $P$, and its coordinates vector is noted $\vec{R} . \Phi^{\prime}=\frac{e^{j k\left\|\vec{R}-\vec{R}^{\prime}\right\|}}{4 \pi\left\|\vec{R}-\vec{R}^{\prime}\right\|}$ is the Green function.

Then, one can establish $[14,15]$ :

$$
\begin{aligned}
K(P) \vec{H}(P) & =\vec{H}_{\mathrm{i}}(P)+\vec{H}_{\mathrm{s}}(P) \\
K(P) & = \begin{cases}0 & \text { if } P \in V \\
1 / 2 & \text { if } P \in S \\
1 & \text { otherwise }\end{cases} \\
\vec{H}_{\mathrm{i}}(P) & =\iiint_{V_{0}} \vec{I}_{H, V}^{\prime} d V^{\prime} \\
\vec{H}_{\mathrm{s}}(P) & =\iint_{S} \vec{I}_{H, S}^{\prime} d S^{\prime}
\end{aligned}
$$

In this expression, the integral kernels are expressed as:

$$
\begin{aligned}
& \vec{I}_{H, V}^{\prime}=j \omega \epsilon \Phi^{\prime} \vec{M}_{\mathrm{i}}^{\prime}+\vec{J}_{\mathrm{i}}^{\prime} \times \vec{\nabla}\left(\Phi^{\prime}\right)+\frac{\rho_{m}^{\prime}}{\mu} \vec{\nabla}\left(\Phi^{\prime}\right) \\
& \vec{I}_{H, S}^{\prime}=-j \omega \mu \Phi^{\prime}\left(\hat{n^{\prime}} \times \vec{E}^{\prime}\right)+\left(\hat{n^{\prime}} \times \vec{H}^{\prime}\right) \times \vec{\nabla}\left(\Phi^{\prime}\right)+\left(\overrightarrow{H^{\prime}} \cdot \hat{n^{\prime}}\right) \vec{\nabla}\left(\Phi^{\prime}\right)
\end{aligned}
$$

In this final expression, $K(P)$ is a function related to the position of point $P$ [15], separating the case $P \in V$, where it highlights extinction theorem, the case $P \in S$, showing the Magnetic Field Integral Equation (MFIE), and the case $P \notin V$ where it is related to the Huygens principle. Note that in case of MFIE, integral of Equation (1d) is a principal value integral [14].

\subsection{PO Approximation}

PO is a method used to calculate the scattered field from an object. It is an asymptotic method based on the fields integral equations and the tangent plane approximation. 
Based on (1), the scattered field can be expressed by introducing currents. Thus, integral kernels are calculated as:

$$
\vec{I}_{H, S}^{\prime}=j \omega \epsilon \Phi^{\prime} \vec{M}^{\prime}+\vec{J}^{\prime} \times \vec{\nabla}\left(\Phi^{\prime}\right)+\frac{j}{\omega \mu}\left(\vec{\nabla} \cdot \vec{M}^{\prime}\right) \vec{\nabla}\left(\Phi^{\prime}\right)
$$

where current densities $\overrightarrow{J^{\prime}}=\hat{n^{\prime}} \times \vec{H}^{\prime}$ and $\vec{M}^{\prime}=-\hat{n}^{\prime} \times \vec{E}^{\prime}$ can be expressed:

$$
\begin{aligned}
\vec{J}^{\prime} & =\hat{n}^{\prime} \times\left(\vec{H}_{\mathrm{i}}\left(P^{\prime}\right)+\vec{H}_{\mathrm{r}}\left(P^{\prime}\right)\right) \\
\vec{M}^{\prime} & =-\hat{n}^{\prime} \times\left(\vec{E}_{\mathrm{i}}\left(P^{\prime}\right)+\vec{E}_{\mathrm{r}}\left(P^{\prime}\right)\right)
\end{aligned}
$$

In PO approximation, reflected fields $\vec{E}_{\mathrm{r}}\left(P^{\prime}\right)$ and $\vec{H}_{\mathrm{r}}\left(P^{\prime}\right)$ are calculated by using Fresnel coefficients [14]. For a perfectly-conducting target, it leads to $\vec{E}_{\mathrm{r}}\left(P^{\prime}\right)=-\vec{E}_{\mathrm{i}}\left(P^{\prime}\right)$ and $\vec{H}_{\mathrm{r}}\left(P^{\prime}\right)=+\vec{H}_{\mathrm{i}}\left(P^{\prime}\right)$. Then, the surface currents are:

$$
\begin{aligned}
\vec{J}^{\prime} & =2 \hat{n}^{\prime} \times \vec{H}_{\mathrm{i}}\left(P^{\prime}\right) \\
\vec{M}^{\prime} & =\overrightarrow{0}
\end{aligned}
$$

Moreover, as PO does not consider creeping waves, another approximation is made: for targets with large dimensions (compared to wavelength), a shadowed zone is considered, over which current vanishes. Thus, integration surface is reduced to illuminated part of surface $S$, called $S_{\text {ill }}$ :

$$
\vec{H}_{\mathrm{S}}(P)=\iint_{S_{\mathrm{ill}}} \vec{I}_{H, S}^{\prime} d S^{\prime}
$$

\subsection{Scattering Problem with 2 Objects: Coupled Integral Equations}

In this part, the problem of scattering by 2 objects is studied. The scenario is illustrated in Figure 2.

Two objects are considered, which volumes and bounding surfaces are $V_{1}, S_{1}$ and $V_{2}, S_{2}$, respectively. Propagation volume is still called $V_{0}$, where $V_{\infty}=V_{1} \cup V_{2} \cup V_{0}$.

Point $P^{\prime}$, of coordinates vector $\vec{R}^{\prime}$, is used for integration. When $P^{\prime}$ is located on surface $S_{1}$ or $S_{2}$, surface element $d S^{\prime}$ is defined, with normal vector $\hat{n}^{\prime}$ pointing towards $V_{0}$. When $P^{\prime}$ is located in a volume, a volume element $d V^{\prime}$ will be defined.

For two objects, Equation (1) is still valid, but $S$ is changed into $S_{1} \cup S_{2}$. Thus, the expressions of the fields are:

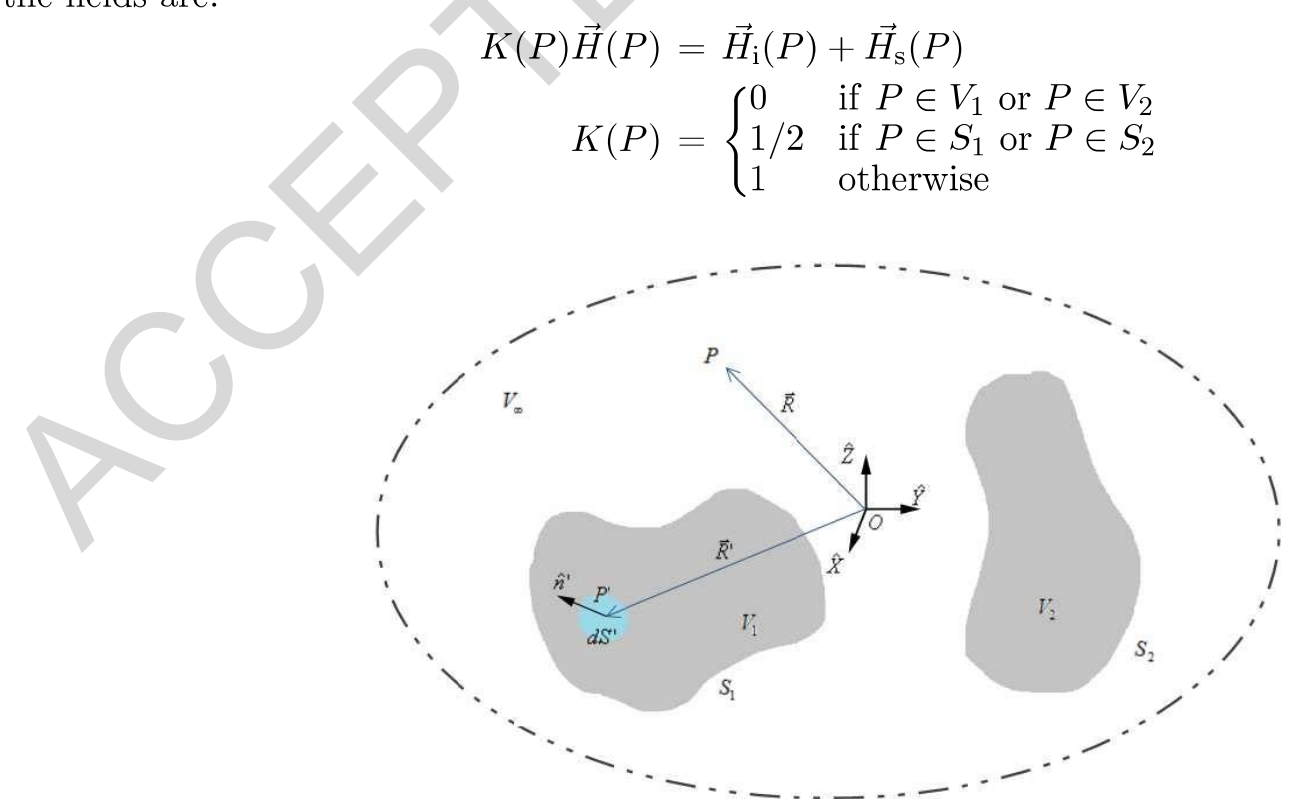

Figure 2. General configuration of scattering problem with 2 objects. 


$$
\begin{aligned}
& \vec{H}_{\mathrm{i}}(P)=\iiint_{V_{0}} \vec{I}_{H, V}^{\prime} d V^{\prime} \\
& \vec{H}_{\mathrm{s}}(P)=\iint_{S_{1}} \vec{I}_{H, S}^{\prime} d S^{\prime}+\iint_{S_{2}} \vec{I}_{H, S}^{\prime} d S^{\prime}
\end{aligned}
$$

Equation (10d) illustrates the superposition theorem, in which surface integrals are coupled, as they depend on total fields $\vec{E}^{\prime}$ and $\vec{H}^{\prime}$, affected by both surfaces. Thus, there are interactions between the two objects.

\subsection{IPO Formulation and Shadowing Phenomenon}

To calculate field scattered by two objects, as the two objects interact with each other, it is necessary to enhance in order to PO take into account the interactions. In IPO, PO is first used to determine surface currents induced by incident field (at iteration 0 ), and then in the iterations to update surface currents (using fields propagating from one object to the other one). Here, the focus is on the way of using PO method at iteration 0.

Let us consider the same case than in previous section (see Figure 2), with an incident field propagating "from $S_{1}$ to $S_{2}$ ": a transmitter (original field source) is inserted at point $P_{\mathrm{i}}$, such that $S_{1}$ is located between $P_{\mathrm{i}}$ and $S_{2}$. Two methods are studied to take into account the shadowing effects in the currents: the classical geometrical shadowing and a novel approach called physical shadowing.

At iteration 0 , for both methods, fields scattered by the two objects are assumed to be uncoupled. Surface currents on $S_{1}$ and the field scattered by $S_{1}$ are determined by the same way for both methods. Since $S_{2}$ is illuminated "after" $S_{1}$, it does not initially interfere on current calculation on $S_{1}$, for each point $P_{1}$ on $S_{1}$, the current is calculated by:

$$
\vec{J}\left(P_{1}\right)=2 \hat{n}\left(P_{1}\right) \times \vec{H}_{\mathrm{i}}\left(P_{1}\right)
$$

On surface $S_{2}$, a shadowing phenomenon occurs, and surface currents on $S_{2}$ are calculated differently depending on using geometrical approach or physical one. Considering a point $P_{2}$ on $S_{2}$, the two methods give different expressions of $\vec{J}\left(P_{2}\right)$.

Geometrical shadowing is based on ray tracing to determine which part of surface $S_{2}$ is shadowed by $S_{1}$. If the ray connecting the points $P_{\mathrm{i}}$ and $P_{2}$ does not cross $S_{1}$, then surface current is calculated from incident field, without taking into account the surface $S_{1}\left(\vec{J}\left(P_{2}\right)=2 \hat{n}\left(P_{2}\right) \times \vec{H}_{\mathrm{i}}\left(P_{2}\right)\right)$. Otherwise, surface current at $P_{2}$ vanishes $\left(\vec{J}\left(P_{2}\right)=\overrightarrow{0}\right), P_{2}$ being considered shadowed by $S_{1}$. To distinguish those cases, an illumination function $I\left(P_{2}\right)$ is introduced, consisting in a Boolean function which mathematically reduces the integration surface. This function can only be determined by a geometrical way, using rays. Then, a general expression of surface current on $S_{2}$ can be obtained:

$$
\begin{aligned}
\vec{J}\left(P_{2}\right) & =\left[2 \hat{n}\left(P_{2}\right) \times \vec{H}_{\mathrm{i}}\left(P_{2}\right)\right] I\left(P_{2}\right) \\
I\left(P_{2}\right) & = \begin{cases}1 & \text { if } P_{2} \text { is illuminated } \\
0 & \text { if } P_{2} \text { is shadowed by } S_{1}\end{cases}
\end{aligned}
$$

Physical shadowing is an interesting approach of shadowing problem, which does not use any geometrical technique to determine the surface currents on shadowed surface. This technique is related to the shadow radiation calculation with PO $[8,9]$. The expression of $\vec{J}\left(P_{2}\right)$ is derived from the coupled magnetic fields integral Equation (10), leading to:

$$
\begin{aligned}
\vec{J}\left(P_{2}\right) & =2 \hat{n}\left(P_{2}\right) \times \vec{H}\left(P_{2}\right) \\
\vec{H}\left(P_{2}\right) & =\vec{H}_{\mathrm{i}}\left(P_{2}\right)+\iint_{S_{1}} \vec{I}_{H, S}^{\prime} d S^{\prime}+\iint_{S_{2}} \vec{I}_{H, S}^{\prime} d S^{\prime}
\end{aligned}
$$

In (13b), the integral over surface $S_{2}$ is assumed to be vanished at iteration 0 , as PO approximation neglects the creeping waves. Also, objects being considered as uncoupled, fields in expression of $I_{H, S}^{\prime}$ for 
$P^{\prime} \in S_{1}$ are independent of $S_{2}$. Thus expression $\iint_{S_{1}} I_{H, S}^{\prime} d S^{\prime}$ corresponds to the magnetic field scattered by surface $S_{1}$ towards point $P_{2}$, which is calculated by PO method and noted $\vec{H}\left(S_{1} \mapsto P_{2}\right)$. Then $\vec{J}\left(P_{2}\right)$ can be expressed as:

$$
\vec{J}\left(P_{2}\right)=2 \hat{n}\left(P_{2}\right) \times\left[\vec{H}_{\mathrm{i}}\left(P_{2}\right)+\vec{H}\left(S_{1} \mapsto P_{2}\right)\right]
$$

Physically, field scattered in forward direction by $S_{1}$ comes to balance the direct incident field at $P_{2}$, expressed as if $S_{1}$ does not exist. By this way, field $\vec{H}\left(S_{1} \mapsto P_{2}\right)$ involves a shadowing effect on surface $S_{2}$, which is physically more rigorous (because closer to the exact coupled integral equations) than that obtained by geometrical shadowing. Moreover, it does not need to use a ray algorithm.

In order to be implemented in IPO, shadowed parts and shadowing parts of the target have to be identified. In [7], the authors use in "IPO shadowing rule" a normal test to avoid any ray tracing: integration is made only for points $P^{\prime}$ satisfying $\hat{n}(P) \cdot\left(\vec{R}-\vec{R}^{\prime}\right)<0$. Thus, using this technique, physical shadowing application is a part of first IPO iteration. Depending on the case, it could also be more interesting to determine analytically the shadowed part and the shadowing part, in order to save computation time (physical shadowing is then iteration 0 , and useless parts of the surfaces can be excluded).

Finally, this theoretical study shows that physical shadowing is a more rigorous approach than geometrical shadowing. Section 3 provides examples of simulations confirming that using physical shadowing in IPO permits to improve currents and RCS predictions.

\section{IMPLEMENTATION AND NUMERICAL RESULTS}

\subsection{Scenario Description: Dihedral Reflector}

The studied case is the bistatic RCS, in $V V$ polarization, of a thin perfectly conducting right-angled dihedral reflector. To reduce the impact of edge diffraction, the $V V$ polarization is selected. However, for the $H H$ polarization, conclusions are similar. This case is particularly interesting in order to exhibit the impact of shadowing approach. Indeed, the influence of iterations will not be very important due to its geometry: the number of iterations necessary for convergence is close to the number of geometrical reflections, which does not exceed 2 in this case. Thus, differences between both shadowing approaches will be highlighted.

The object is depicted in Figure 3(a) and parameters common to each simulation are given in Table 1. It consists in two orthogonal plates with infinitely small thickness, noted $S_{X}$ and $S_{Z}$. The two sides of each plate are considered apart from each other and noted among their normal directions $: \hat{n}\left(S_{X}^{+}\right)=+\hat{Z}, \hat{n}\left(S_{X}^{-}\right)=-\hat{Z}, \hat{n}\left(S_{Z}^{+}\right)=+\hat{X}$ and $\hat{n}\left(S_{Z}^{-}\right)=-\hat{X}$. Incidence and observation points, noted $P_{\mathrm{i}}$ and $P_{\text {obs }}$ respectively, are located in plane $(\hat{X} O \hat{Z})$ by their elevation angles $\theta_{\mathrm{i}}$ and $\theta_{\mathrm{s}}$. Oriented angles are defined in clockwise direction.

Simulations are run with a meshing around $\lambda / 8$, as in MoM, in order to have coherent comparisons, but IPO can be used with bigger meshes without loss of accuracy.

Table 1. Global simulation parameters.

\begin{tabular}{|c|c|c|c|}
\hline Parameter & Notation & Justification & Value \\
\hline frequency & $F$ & X band & $10 \mathrm{GHz}$ \\
\hline planes length & $L$ & $8 \lambda$ & $240 \mathrm{~mm}$ \\
\hline planes thickness & $\epsilon$ & no thickness & 0 \\
\hline depth among $\hat{Y}$ & $D_{y}$ & $8 \lambda$ & $240 \mathrm{~mm}$ \\
\hline points per length & $N_{\text {pts }}$ & meshing around $\lambda / 8$ & 63 \\
\hline number of iterations & $N_{\text {iter }}$ & convergence & 2 \\
\hline
\end{tabular}




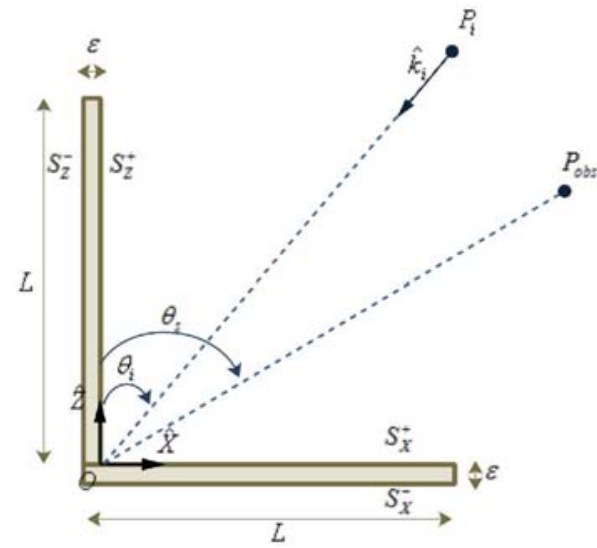

(a)

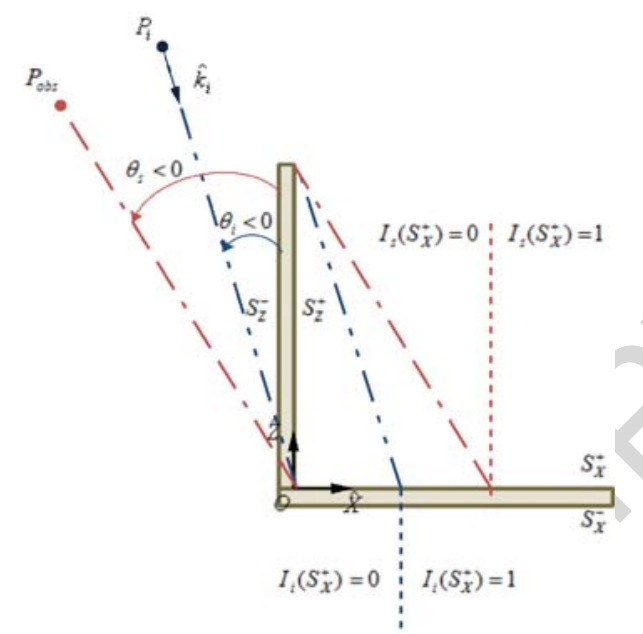

(b)

Figure 3. Geometrical configuration and illustration of geometrical shadowing. (a) Dihedral reflector configuration. (b) Particular case showing geometrical shadowing at both incidence and observation.

Shadowing effects (see Section 3.2) can be considered both from the transmitter (when the dihedral is partly illuminated due to shadowing from other parts) and towards the receiver (when a part of dihedral cannot be seen by the observation point without passing through another part). Therefore two different scenarii are proceeded: one with a fix incidence and a varying observation, showing the effect of shadowing towards receiver; and one with several incidence directions, showing cases of shadowing from transmitter.

The relative norm 2 error is used to estimate the validity of the different results. It is defined, for a set of $N$ components of $\vec{V}$, in comparison to a reference set $\vec{V} R E F$, by:

$$
\begin{aligned}
\epsilon^{R E F}(\vec{V}) & =\frac{\left\|\vec{V}-\vec{V}^{R E F}\right\|}{\left\|\vec{V}^{R E F}\right\|} \\
\text { where }\|\vec{Y}\| & =\sqrt{\sum_{x=1}^{N}\left|Y_{x}\right|^{2}}
\end{aligned}
$$

$Y_{x}, x=1, \ldots, N$, representing the $N$ elements of any vector $\vec{Y}$.

\subsection{Implementation}

In order to explain the implementation of both kind of shadowing from the transmitter and towards receiver, let us consider the case of an incidence angle $\theta_{\mathrm{i}} \in(-45 ; 0)^{\circ}$ and observation angle $\theta_{\mathrm{s}} \in\left(-45^{\circ} ; \theta_{\mathrm{i}}\right)$ (see Figure 3(b)). These shadowing effects are described below, their consideration is illustrated in Figure 4, showing the implementation of IPO algorithm.

From incidence point $P_{i}$, a shadowing effect is to be considered for surface $S_{X}^{+}$. The geometrical shadowing needs to calculate an illumination function $\left(I_{\mathrm{i}}\left(S_{X}^{+}\right)\right)$. This function is a Boolean function separating the part of the surface where the current is calculated by PO approximation $(\vec{J}=$ $\left.2 \hat{n}\left(S_{X}^{+}\right) \times \vec{H}_{\mathrm{i}}\left(S_{X}^{+}\right)\right)$from the part where current is forced to zero $(\vec{J}=\overrightarrow{0})$. In case of physical shadowing, shadowing effect is applied by considering a field propagating from $S_{Z}^{-}$in its forward direction balancing incident field $\left(\vec{J}=\hat{n} \times\left[\vec{H}_{\mathrm{i}}+\vec{H}\left(S_{Z}^{-} \mapsto S_{X}^{+}\right)\right]\right)$.

The receiver (after iterations of IPO), located on $P_{\text {obs }}$ cannot directly observe the whole $S_{X}^{+}$surface. Geometrical approach consists in considering another boolean function $\left(I_{\mathrm{S}}\left(S_{X}^{+}\right)\right)$, separating the part 


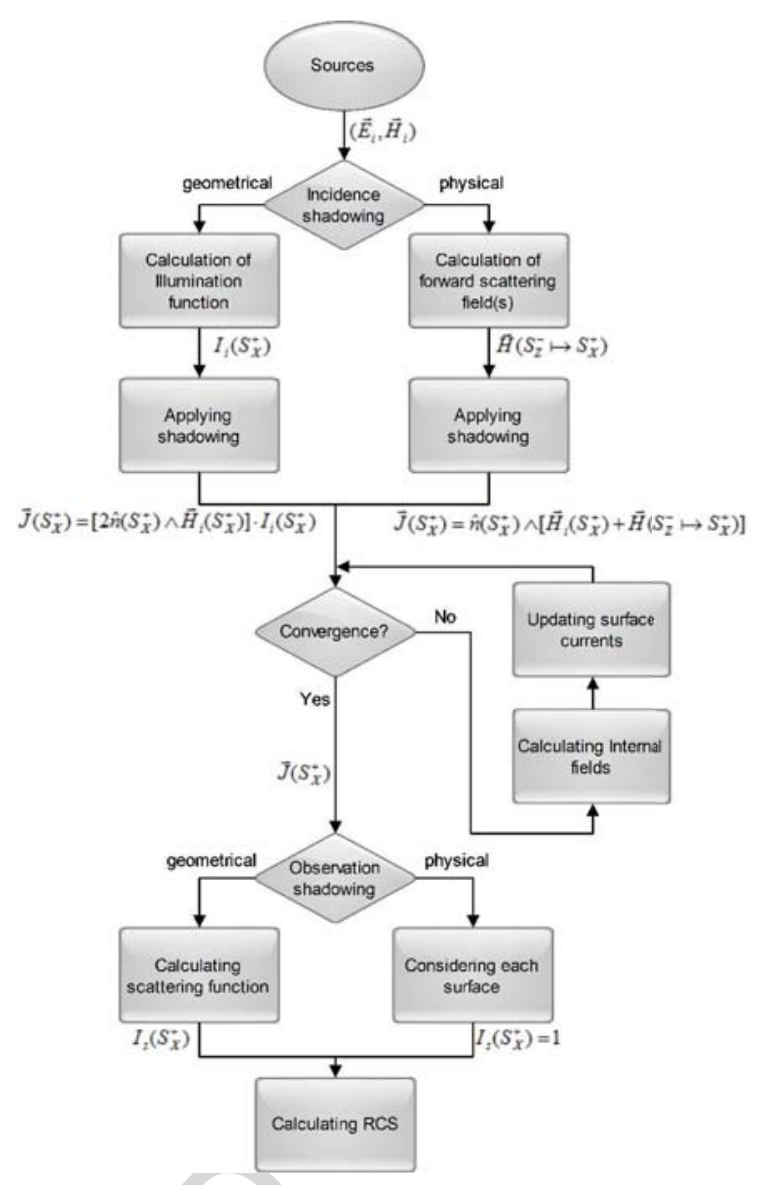

Figure 4. IPO algorithm with different cases of shadowing.

of the surface that can be directly observed from the part hidden by $S_{Z}$ plane, where currents will be forced to zero for radiation. Using physical shadowing approach at observation simply leads to consider each surface current in radiation, following the Huygens principle, fields induced by currents from $S_{Z}^{+}$ being balancing those from $S_{X}^{+}$.

Note that in case of more complex geometries a third case of shadowing effect can be considered inside the cavity.

\subsection{Numerical Results: Shadowing toward Receiver}

First, a simulation has been set in order to show the differences between both shadowing approaches at observation. In this case, an incidence from the dihedral's bisector $\left(\theta_{i}=+45^{\circ}\right)$ is considered, and observation angle varies $\left(\theta_{s} \in[-180 ; 180]^{\circ}\right)$. Thus, depending on the observation angle, the whole dihedral or a part of it can be seen at the observation point. In this case, there is no shadowing effect at incidence, since the whole $S_{X}^{+}$and $S_{Z}^{+}$surfaces are illuminated, and thus physical shadowing and geometrical shadowing are the same.

Figure 5(a) shows the obtained results, comparing IPO method with physical shadowing, IPO with geometrical shadowing and MoM. Figure 5(b) plots a zoom of this result in the most interesting zone of observation. Also errors in different zones are given in Table 2.

These results can be analyzed by splitting up the observation domain in different zones:

- for $\theta_{\mathrm{S}} \in(0 ; 90)^{\circ}$, both $S_{X}^{+}$and $S_{Z}^{+}$surfaces are fully seen by the receiver, and $S_{X}^{-}$and $S_{Z}^{-}$are unseen. In this case, there is no shadowing effect and geometrical shadowing and physical shadowing give exactly the same results. These results are close to MoM reference results, especially around the 


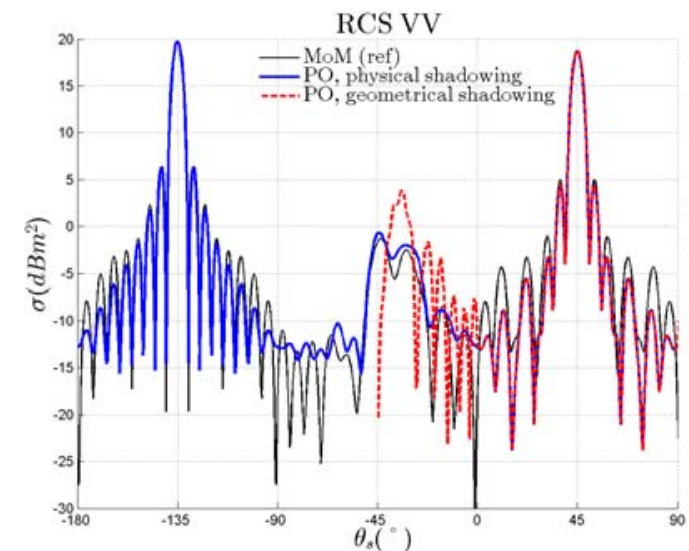

(a)

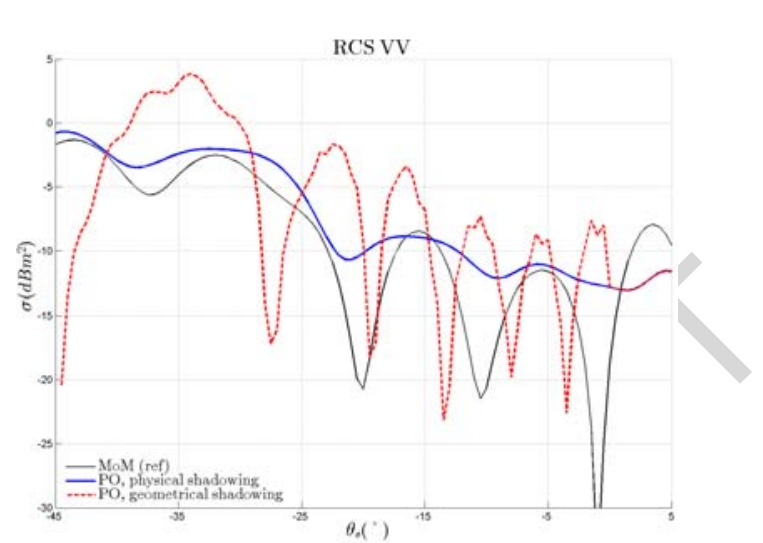

(b)

Figure 5. Results for shadowing towards receiver. Case of the dihedral reflector for $\theta_{i}=+45^{\circ}$; comparing MoM, IPO with physical shadowing and IPO with geometrical shadowing; see Table 1 for general parameters. (a) RCS $V V$ versus $\theta_{s}$ for $\theta_{s}$ in $[-180 ; 90]^{\circ}$. (b) Enlarged details for $\theta_{s}$ in $[-45 ; 5]^{\circ}$.

Table 2. Bistatic RCS $V V$ error per zone, related to results in Figure 5; comparing IPO with physical shadowing and IPO with geometrical shadowing to MoM.

\begin{tabular}{|c|c|c|c|c|}
\hline$\epsilon^{\mathrm{MoM}}(\sigma)(\%)$ & $(0 ; 90)^{\circ}$ & $(-45 ; 0)^{\circ}$ & $(-90 ;-45)^{\circ}$ & $(-180 ;-90)^{\circ}$ \\
\hline IPO, physical shadowing & 3.7 & 32 & 34 & 0.61 \\
\hline IPO, geometrical shadowing & 3.7 & 199 & - & - \\
\hline
\end{tabular}

specular direction $\theta_{\mathrm{i}}=+45^{\circ}$, and differences are mainly due to the non consideration of edge diffraction by $\mathrm{PO}$;

- for $\theta_{\mathrm{s}} \in[-45 ; 0)^{\circ}$ (see enlarged details Figure 5(b)), surfaces $S_{X}^{-}$and $S_{Z}^{+}$cannot be seen, $S_{Z}^{-}$is fully seen (but not illuminated), and surface $S_{X}^{+}$is partially shadowed by $S_{Z}$ plate. Thus only a part of $S_{X}^{+}$is considered in geometrical shadowing, while in physical shadowing approach, field from $S_{Z}^{+}$comes to balance field from the whole $S_{X}^{+}$plane. Differences can be observed between both techniques, and physical shadowing globally leads to results closer to MoM;

- for $\theta_{\mathrm{s}} \in[-90 ;-45)^{\circ}$, surfaces $S_{X}^{-}$and $S_{Z}^{+}$cannot be seen, $S_{Z}^{-}$is fully seen, and surface $S_{X}^{+}$is still shadowed by $S_{Z}$ plate. The difference is that $S_{X}^{+}$is now considered fully shadowed by $S_{Z}$ in geometrical shadowing, while physical shadowing still considers that the field scattered from $S_{Z}^{+}$ balances field from the whole $S_{X}^{+}$surface. Thus geometrical shadowing leads to a non existent scattered field, and a RCS equal to $0 \mathrm{~m}^{2}\left(-\infty \mathrm{dBm}^{2}\right)$, while IPO with physical shadowing still matches relatively well the reference;

- for $\theta_{\mathrm{S}} \in(-180 ;-90)^{\circ}$, surfaces $S_{X}^{-}$and $S_{Z}^{-}$, where currents vanish, are directly seen at observation point, while illuminated surfaces $S_{X}^{+}$and $S_{Z}^{+}$are hidden. For geometrical shadowing, for the same reason than previous zone, RCS vanishes (to be rigorous, except at the single point $\theta_{\mathrm{s}}=-135^{\circ}$, GO being able to calculate scattered field in the exact forward scattering direction). For physical shadowing, this case ends up to typical forward scattering using PO approximation $[8,9]$, and the calculated RCS is very close to the reference.

Note that the same observations can be made in the other sense $\left(\theta_{\mathrm{s}} \in[90 ; 180)^{\circ}\right)$ by symmetry. Moreover, a discontinuity can be observed for geometrical shadowing at $\theta_{\mathrm{s}}=0^{\circ}$ (and also at $\theta_{\mathrm{s}}=+90^{\circ}$ ), obvious in Figure $5(\mathrm{~b})$, due to the fact that $I_{\mathrm{S}}\left(S_{Z}^{+}\right)$is suddenly forced to zero. This non physical discontinuity is avoided by the use of physical shadowing. 
In conclusion, using physical shadowing at observation in IPO gives more complete and accurate results. Indeed, it leads to a more general result (permitting observation around the forward scattering direction), it permits to suppress any discontinuity due to the geometry, and, in zones where it differs from geometrical approach of shadowing effect, the results obtained with physical shadowing are closer to reference method.

\subsection{Numerical Results: Shadowing from the Transmitter}

This section shows series of results for $\theta_{\mathrm{i}} \in[-45 ; 0)^{\circ}$. In such a case, as seen previously, a shadowing effect is to be considered from the transmitter for surface $S_{X}^{+}$. Observation is made over $\theta_{\mathrm{s}} \in[0 ; 90]^{\circ}$ so that influence of shadowing at observation is weak: for geometrical shadowing, no ray boucing is needed towards the receiver, and the only difference with physical shadowing at observation is the field scattered by $S_{Z}^{-}$in forward direction, which has a slight influence on results. Also, surface current $\vec{J}\left(S_{X}^{+}\right)$is observed, for both cases of shadowing, in comparison with MoM surface currents behaviors.

Figures 6(a), 6(b) and 6(c) show results of bistatic RCS $V V$ and surface currents on $S_{X}^{+}$for incidence angles $\theta_{\mathrm{i}}=-10^{\circ}, \theta_{\mathrm{i}}=-20^{\circ}$ and $\theta_{\mathrm{i}}=-30^{\circ}$, respectively. Tables 3 and 4 present the errors calculated for both methods, on RCS and currents, respectively.
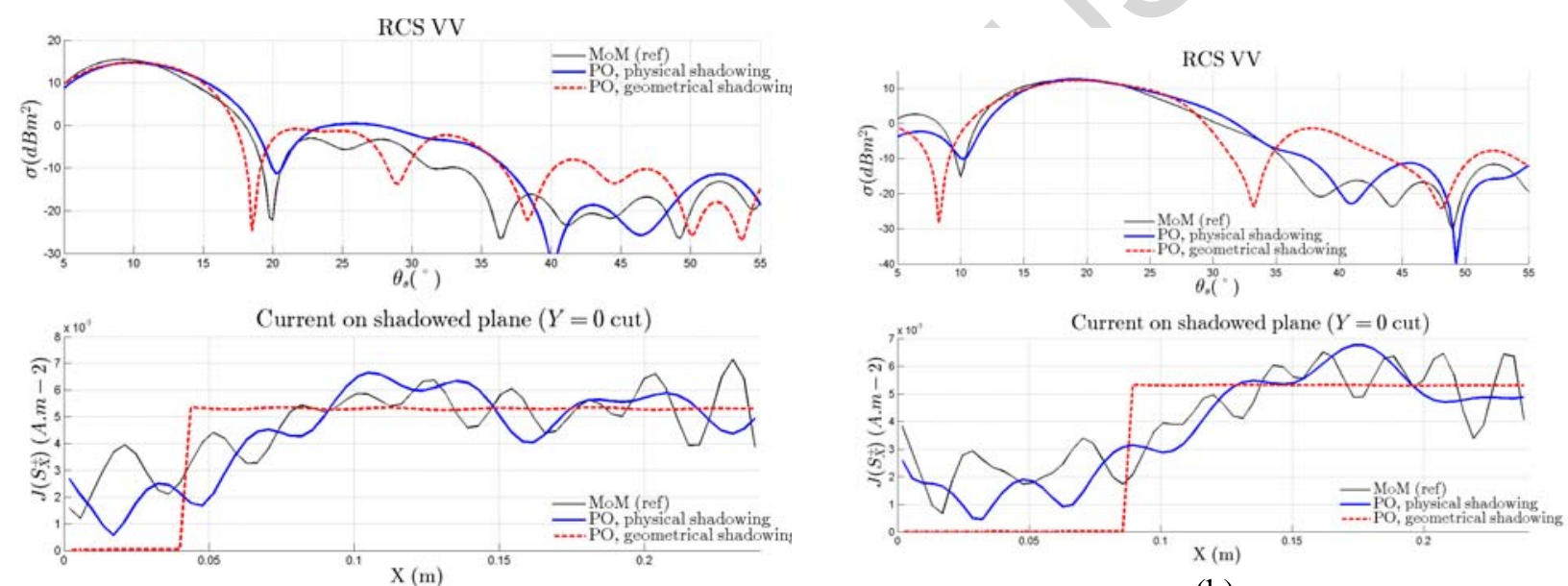

(a)

(b)

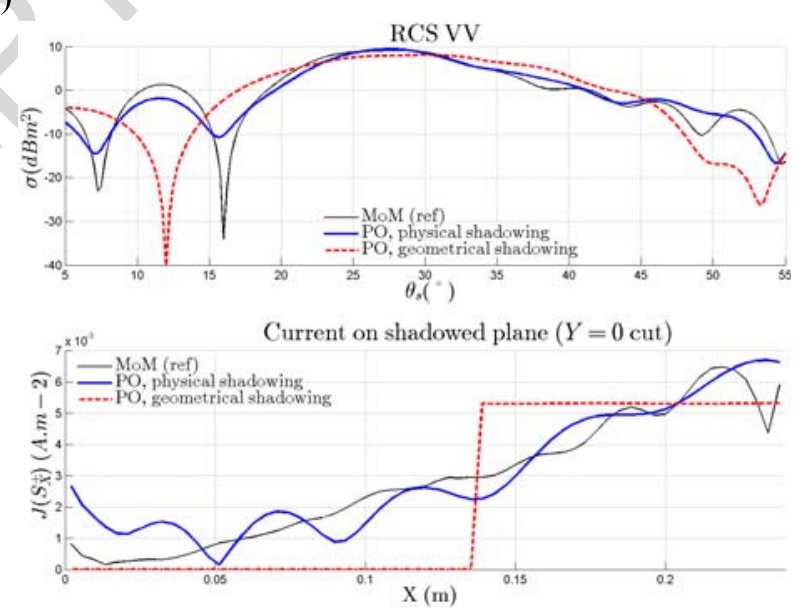

(c)

Figure 6. RCS in $V V$ polarization versus $\theta_{s}$ and surface current versus $X$ for different values of incidence angle: (a) $\theta_{i}=-10^{\circ}$; (b) $\theta_{i}=-20^{\circ}$; (c) $\theta_{i}=-30^{\circ}$; comparing MoM, PO with physical shadowing and PO with geometrical shadowing; see Table 1 for general parameters. 
Table 3. RCS $V V$ error per incidence angle, related to results Figures 6 and 7; comparing IPO with physical shadowing and IPO with geometrical shadowing to MoM.

\begin{tabular}{|c|c|c|c|c|}
\hline$\epsilon^{\mathrm{MoM}}(\sigma)(\%)$ & $\theta_{\mathrm{i}}=-10^{\circ}$ & $\theta_{\mathrm{i}}=-20^{\circ}$ & $\theta_{\mathrm{i}}=-30^{\circ}$ & $\theta_{\mathrm{i}}=-45^{\circ}$ \\
\hline physical shadowing & 20 & 14 & 13 & 39 \\
\hline geometrical shadowing & 18 & 14 & 31 & - \\
\hline
\end{tabular}

Table 4. Surface current error per incidence angle, related to results Figures 6 and 7; comparing IPO with physical shadowing and IPO with geometrical shadowing to MoM.

\begin{tabular}{|c|c|c|c|c|}
\hline$\epsilon^{\mathrm{MoM}}(J)(\%)$ & $\theta_{\mathrm{i}}=-10^{\circ}$ & $\theta_{\mathrm{i}}=-20^{\circ}$ & $\theta_{\mathrm{i}}=-30^{\circ}$ & $\theta_{\mathrm{i}}=-45^{\circ}$ \\
\hline physical shadowing & 24 & 24 & 21 & 42 \\
\hline geometrical shadowing & 29 & 39 & 44 & - \\
\hline
\end{tabular}
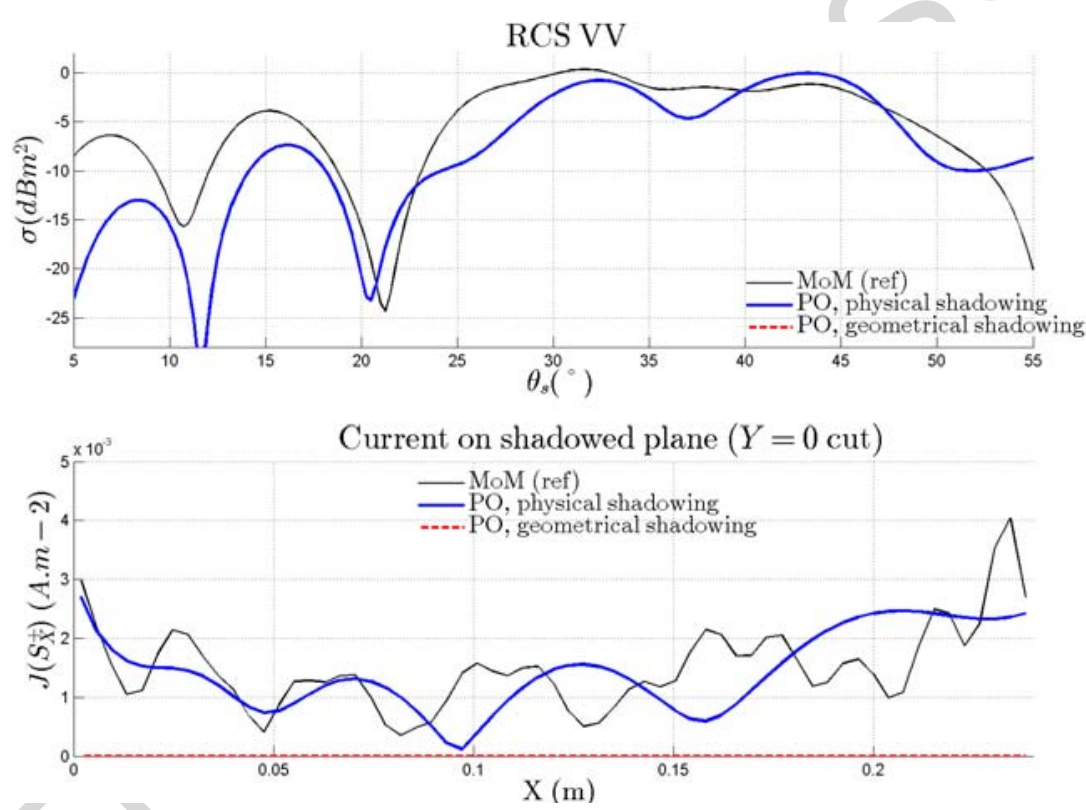

Figure 7. RCS in $V V$ polarization versus $\theta_{s}$ and surface current versus $X$ for extreme case of $\theta_{\mathrm{i}}=-45^{\circ}$; comparing MoM, PO with physical shadowing and $\mathrm{PO}$ with geometrical shadowing (vanishing currents and RCS); see Table 1 for general parameters.

In such cases, using geometrical shadowing at iteration 0 consists in determining $I_{\mathrm{i}}\left(S_{X}^{+}\right)$and multiplying by the obtained Boolean, the current calculated by PO approximation from the incident field ; physical shadowing consists in adding the field from $S_{Z}^{-}$to the incident field.

Observing the current progression among $X$ axis permits to confirm that physical shadowing is physically more realistic than geometrical technique. Indeed, the geometrical model presents a discontinuous behavior, which is not physical, and leads to a result less close to the one obtained by MoM. If the current looks more realistic using physical shadowing for any of the 3 incidence angles considered here, improvement (compared to $\mathrm{MoM}$ ) is more obvious for angles implying more shadowing effects.

In the different cases of Figure 6 , RCS versus $\theta_{\mathrm{S}}$ is near a cardinal sine shape. This behavior can be simply explained based on IPO with geometrical shadowing. Indeed in this case, the RCS is the one of the geometrically illuminated part of $S_{X}^{+}$plane (the part in which $I_{\mathrm{i}}\left(S_{X}^{+}\right)=1$ ), modified by the 
iterating process (which is not very influent in the present case). The general formula of RCS $V V$ of a rectangular plate of dimensions $L_{X}$ by $L_{Y}$ is provided in (16), and justifies the sinc shape.

$$
\sigma=\frac{4 \pi\left(L_{X} L_{Y}\right)^{2}}{\lambda^{2}} \operatorname{sinc}^{2}\left(\frac{k L_{X}}{2}\left[\sin \left(\theta_{\mathrm{i}}\right)-\sin \left(\theta_{\mathrm{s}}\right)\right]\right) \cos ^{2}\left(\theta_{\mathrm{i}}\right)
$$

Using physical shadowing permits a better consideration of the distortion of the sinc shape, which is observed on the MoM results. Thus, results obtained by IPO are better with physical shadowing than with geometrical shadowing, which is more obvious for incidence angle far from zero.

Last, case of an incidence $\theta_{\mathrm{i}}=-45^{\circ}$ shows an extreme case highlighting interest of using physical shadowing. Figure 7 shows the corresponding results. With such an angle, surface $S_{X}^{+}$is geometrically fully shadowed $\left(I_{i}\left(S_{X}^{+}\right)=0\right.$ all along the surface). Then geometrical shadowing ends up in vanishing currents. On the other hand, physical shadowing gives surface current quite close to the MoM one. Then the non zero currents obtained by MoM and IPO with physical shadowing leads to an existing signature, whereas RCS calculated by IPO with geometrical shadowing ends up in a RCS of $0 \mathrm{~m}^{2}\left(-\infty \mathrm{dBm}^{2}\right)$.

As seen previously, in such a case geometrical shadowing considers that $S_{X}^{+}$is fully shadowed by $S_{Z}$ plane. On the other hand, using physical shadowing consists in adding the field from $S_{Z}^{-}$to the incident field, which does not end up in a current constantly equal to zero. Relatively close to the MoM current, this result shows a particular case where geometrical shadowing cannot permit to calculate RCS, while IPO with physical shadowing does, with quite a good accuracy.

\section{CONCLUSION}

This paper provides a comparison of two shadowing techniques used in generalized IPO method (applied to arbitrary geometries): geometrical shadowing and physical shadowing (interpreting shadowing as interference of shadow radiation on incident wave). Based on MFIE applied to electromagnetic scattering by coupled objects, it has been demonstrated that physical shadowing is more rigorous than the classically used geometrical shadowing.

The results show an improvement of RCS prediction by IPO while using physical shadowing instead of the classical geometrical approach, both at incidence and observation. Results obtained after iterations are closer to reference results, and physical shadowing is more complete, as it permits to treat cases of incidence and observation where geometrical shadowing is not able to furnish a result.

\section{ACKNOWLEDGMENT}

This work is supported by the French Defense agency DGA (Direction Générale de l'Armement).

\section{REFERENCES}

1. Obelleiro-Basteiro, F., J. L. Rodriguez, and R. J. Burkholder, "An iterative physical optics approach for analyzing the electromagnetic scattering by large open-ended cavities," IEEE Trans. Antennas Propagat., Vol. 43, No. 4, 356-361, 1995.

2. Hémon, R., P. Pouliguen, H. He, J. Saillard, and J. F. Damiens, "Computation of EM field scattered by an open-ended cavity and by a cavity under radome using the iterative physical optics," Progress In Electromagnetics Research, Vol. 80, 77-105, 2008.

3. Burkholder, R. J. and T. Lundin, "Forward-backward iterative physical optics algorithm for computing the RCS of open-ended cavities," IEEE Trans. Antennas Propagat., Vol. 53, No. 2, 793-799, 2005.

4. Burkholder, R. J., C. Tokgöz, C. J. Reddy, W. O. Coburn, and P. H. Pathak, "Iterative physical optics: Its not just for cavities anymore [EM wave propagation]," IEEE Trans. Antennas Propagat., Vol. 1A, 18-21, 2005.

5. Choi, S. H., D. W. Seo, and N. H. Myung, "Scattering analysis of open-ended cavity with inner object," Journal of Electromagnetic Waves and Applications, Vol. 21, No. 12, 1689-1702, 2007. 
6. Obelleiro, F., J. Campos Nio, J. L. Rodriguez, and A. G. Pino, "A segmented approach for computing the electromagnetic scattering of large and deep cavities," Progress In Electromagnetics Research, Vol. 19, 129-145, 1998.

7. Burkholder, R. J., C. Tokgöz, C. J. Reddy, and W. O. Coburn, "Iterative physical optics for radar scattering predictions," Applied Computational Electromagnetics Society (ACES), Vol. 24, No. 2, 2009.

8. Kubické, G., Y. A. Yahia, C. Bourlier, N. Pinel, and P. Pouliguen, "Bridging the gap between the Babinet principle and the physical optics approximation: Scalar problem," IEEE Trans. Antennas Propagat., Vol. 59, No. 12, 4725-4732, 2011.

9. Kubické, G., C. Bourlier, M. Delahaye, C. Corbel, N. Pinel, and P. Pouliguen, "Bridging the gap between the Babinet principle and the Physical Optics approximation: Vectorial Problem," Radio Science, Vol. 48, No. 5, 573-581, 2013.

10. Ufimtsev, P. Y., "Blackbodies and shadow radiation," Soviet Journal of Communications, Technology and Electronics, Vol. 35, No. 5, 108-116, Translation from Russian by Scripta Technica, Inc., 1990.

11. Ufimtsev, P. Y., Fundamentals of the Physical Theory of Diffraction, John Wiley and Sons, New Jersey, 2007.

12. Siegel, K. M., "Bistatic radars and forward scattering," Aero Electronics Nat. Conf. Proc., 286-290, Dayton, Ohio, 1958.

13. Glaser, J. I., "Bistatic RCS of complex objects near forward scatter," IEEE Trans. Antennas Propagat., Vol. 21, No. 1, 70-78, 1985.

14. Stratton, J. A., Electromagnetic Theory, McGraw-Hill, 1941.

15. Poggio, A. J. and E. K. Miller, Integral Equations Solutions of Three-dimensional Scattering Problems, Computer Techniques for Electromagnetics, R. Mittra, Pergamon Press, 1973. 